\title{
Repercussões do transtorno disfórico pré-menstrual entre universitárias
}

\author{
Effects of premenstrual dysphoric disorder among college students
}

\author{
Valéria Conceição Passos de Carvalho ${ }^{1}$, Amaury Cantilino², Nathália Machado Porto Carreiro ${ }^{3}$, Luciene \\ Fontes de Sá ${ }^{3}$, Everton Botelho Sougey ${ }^{4}$
}

${ }^{1}$ Doutoranda, Programa de Pós-Graduação em Neuropsiquiatria e Ciências do Comportamento, Universidade Federal de Pernambuco (UFPE), Recife, PE. Professora assistente, Universidade Católica de Pernambuco (UNICAP), Recife, PE. Professora assistente, Faculdade Integrada do Recife (FIR), Recife, PE. ${ }^{2}$ Doutor, Neuropsiquiatria e Ciências do Comportamento. Coordenador, Programa de Saúde Mental da Mulher, Núcleo de Pesquisa em Transtornos Afetivos, Hospital das Clínicas, UFPE. ${ }^{3}$ Especialização, Fisioterapeuta, FIR. ${ }^{4}$ Pós-Doutor, Universidade Livre de Bruxelas, Bruxelas, Bélgica. Coordenador, Pós-Graduação em Neuropsiquiatria e Ciências do Comportamento, UFPE. Este estudo foi realizado no Programa de Pós-Graduação, Neuropsiquiatria e Ciências do Comportamento, Universidade Federal de Pernambuco (UFPE), Recife, PE.

\section{Resumo}

Introdução: O transtorno disfórico pré-menstrual (TDPM) é uma variante mais grave da síndrome pré-menstrual que apresenta a oscilação do humor como fator mais perturbador e debilitante, repercutindo na qualidade de vida das mulheres em idade reprodutiva. O objetivo deste estudo foi identificar as principais repercussões físicas e emocionais causadas pela TDPM entre universitárias de Fisioterapia.

Método: Estudo do tipo corte transversal, descritivo e analítico, realizado no período de agosto a dezembro de 2008 em 259 universitárias. Os instrumentos utilizados para a realização desta pesquisa foram: um questionário contendo questões socioeconômicas, o Questionário de Sintomas Menstruais (Menstrual Symptom Questionnaire, MSQ), a escala de autoavaliação de síndrome de transtorno pré-menstrual de Steiner e o Questionário de Autoavaliação da Escala de Hamilton para Depressão. A análise estatística foi descritiva e analítica, sendo utilizado o teste qui-quadrado ou o teste exato de Fisher, sendo que todas as conclusões foram tomadas ao nível de significância de $5 \%$.

Resultados: Encontramos alta prevalência de repercussões somáticas e emocionais. Através dos sinais e sintomas pesquisados pelo MSQ entre as portadoras de TDPM, observou-se uma associação do transtorno com os sintomas depressivos $(p=0,048)$. Os demais sintomas como ansiedade, labilidade de humor, sintomas somáticos, habilidade para realizar tarefas e dor não apresentaram correlação.

Conclusão: O TDPM provocou desconforto físico e emocional e revelou uma correlação significativa com os sintomas depressivos, sugerindo tratar-se de uma expressão clínica de transtorno de humor.

Descritores: Transtorno disfórico pré-menstrual, ciclo menstrual, saúde da mulher

\begin{abstract}
Background: Maternal premenstrual dysphoric disorder (PMDD) is a severe variant of premenstrual syndrome in which mood swings are the most disturbing and debilitating, affecting the quality of life of women of reproductive age. The objective of this study was to identify the main physical and emotional manifestations of PMDD among undergraduate students of Physical Therapy.

Methods: A cross-sectional, descriptive, analytical study was carried between August and December 2008, with 259 undergraduate students. The following instruments were used: a questionnaire covering social and economic data; the Menstrual Symptom Questionnaire (MSQ); Steiner's selfrated premenstrual syndrome questionnaire; and the Hamilton Self-Rating Depression Questionnaire. Descriptive and analytical statistical analysis was performed using the chi-square test or Fisher's exact test. Significance was set at 5\%.

Results: A high prevalence of physical and emotional symptoms was found. Among the signs and symptoms assessed by MSQ, only depression showed a significant correlation with PMDD $(p=0.048)$. Other symptoms, such as anxiety, mood lability, physical symptoms, the ability to perform daily tasks, and pain, were not correlated with PMDD.

Conclusion: PMDD caused physical and emotional distress among the women assessed and was significantly correlated with depression. These findings suggest that PMDD should be classified as a clinical manifestation of mood disorders.
\end{abstract}

Keywords: Premenstrual dysphoric disorder, menstrual cycle, women's health.

Correspondência:

Valéria Conceição Passos de Carvalho, Rua Guedes Pereira, 77/401, CEP 52060-150, Bairro Casa Amarela, Recife, PE. E-mail: valeriapassos@gmail.com Não há conflitos de interesse associados à publicação deste artigo. 


\section{Introdução}

O ciclo menstrual (CM) dura, em média, 28 dias, podendo ser dividido em três fases distintas: folicular, ovulatória e lútea. A fase folicular inicia-se no primeiro dia da menstruação e dura entre 9 e 23 dias, a fase ovulatória pode durar até 3 dias e a fase lútea vai do fim da ovulação até o início do fluxo menstrual. Estas fases são caracterizadas por um perfil específico de concentração sérica dos hormônios sexuais ${ }^{1}$.

Desta forma, o período pré-menstrual é um período de vulnerabilidade para o aparecimento de sintomas físicos e psíquicos que tipicamente se apresentam 1 semana antes da menstruação e aliviam com o início do fluxo menstrual. Segundo a estimativa de pesquisas epidemiológicas, 75\% das mulheres em idade reprodutiva experimentam alguns sintomas atribuídos à fase pré-menstrual do ciclo. Mais de 100 sintomas físicos e psicológicos vêm sendo reportados, mas muitas mulheres são capazes de administrá-los através de mudanças no estilo de vida e de terapias conservadoras ${ }^{2}$.

A síndrome disfórica pré-menstrual ou transtorno disfórico pré-menstrual (TDPM) é uma variante da síndrome prémenstrual, mais grave, que apresenta oscilação intensa de humor como fator mais perturbador e debilitante no complexo de sintomas existentes. Sua etiologia não é bem definida, pois ela é influenciada por fatores hormonais, familiares, ambientais e socioculturais, não apresentando necessariamente a sintomatologia física. Esse quadro, entretanto, influencia de forma importante as atividades rotineiras e de trabalho das mulheres ${ }^{2-4}$.

Os sintomas do TDPM podem aparecer desde a menarca até a menopausa e encontram-se presentes na vida das mulheres ao longo de todo o período reprodutivo, sendo muitas vezes interpretados como sintomas de um transtorno imaginário e resultantes da cada vez mais estressante vida moderna. Por outro lado, inúmeras mulheres relatam apresentar um aumento da gravidade e da duração dos sintomas com a proximidade da menopausa, o que repercute na sua qualidade de vida ${ }^{3}$.

A indefinição sobre o conceito de TDPM explica a multiplicidade de escalas e critérios diagnósticos existentes para esse transtorno, não havendo ainda uma metodologia padrão para sua avaliação. A validação de escalas dessa síndrome mal delimitada está incompleta, e as escalas existentes tentam estabelecer um ou mais padrões de mudança e de intensidade dos sintomas ao longo do ciclo menstrual ${ }^{5}$.

O diagnóstico diferencial entre a síndrome da tensão prémenstrual (TPM) e o TDPM pode ser difícil, pois não existe nenhum exame laboratorial que possa servir como marcador biológico de uma ou outra síndrome. Entretanto, a maioria dos ginecologistas ou psiquiatras elabora o seu diagnóstico através da avaliação dos sintomas exacerbados no período menstrual, sejam eles sintomas físicos ou sintomas depressivos ${ }^{6}$.

A identificação precoce e o tratamento adequado do TDPM reduzem a probabilidade de que ele se torne crônico e recorrente. Ainda há uma controvérsia quanto às formas de intervir neste transtorno, porém a reeducação alimentar, a administração de medicamentos (psicofármacos e contra- ceptivos) e a atividade física têm demonstrado uma contribuição positiva para as portadoras do TDPM ${ }^{1,4}$.

O TDPM apresenta como principal característica sua recorrência clínica durante a fase lútea e sintomas de humor e comportamentais, dentre os quais a depressão, ansiedade, labilidade afetiva, tensão, irritabilidade e distúrbios do sono são os mais frequentes. Além disso, causa um forte comprometimento do funcionamento social e ocupacional do indivíduo, que geralmente pioram com a aproximação da menstruação e usualmente cessam de forma imediata com o início do fluxo menstrual ${ }^{7}$.

O presente projeto tem como objetivo identificar as principais repercussões psicossomáticas causadas pelo TDPM entre as universitárias, descrevendo a sua prevalência e investigando sua associação com fatores socioeconômicos, demográficos e comportamentais.

\section{Materiais e método}

Estudo de corte transversal, descritivo e analítico. Foi desenvolvido na Faculdade Integrada do Recife (FIR), Recife (PE), no período de agosto a dezembro de 2008. Aprovado pelo Comitê de Ética em Pesquisa com Seres Humanos da Universidade Federal de Pernambuco (UFPE), Recife (PE) (CAAE: 0001.0.100.172-08).

A amostra foi composta por 259 acadêmicas devidamente matriculadas no curso de Fisioterapia da FIR mediante a assinatura do termo de consentimento livre e esclarecido. Foram excluídas mulheres com amenorreia (não menstruam, incluindo gravidez) e mulheres que apresentavam ciclos irregulares (menor que 25 dias ou maior que 35 dias).

A coleta de dados foi realizada através de uma abordagem das universitárias na sala de aula, onde, primeiramente, foram explicados os objetivos da pesquisa e, depois, distribuídos os instrumentos do estudo. Foi garantido o sigilo das informações contidas, de forma que as universitárias não fossem identificadas.

Os instrumentos utilizados para a realização desta pesquisa foram: um questionário que abordava questões socioeconômicas, história clínica, hábitos de vida e escalas específicas para o fim da pesquisa, contendo questões fechadas e estruturadas em tópicos para facilitar o ordenamento lógico das idéias; o Questionário de Sintomas Menstruais (Menstrual Symptom Questionnaire, MSQ), contendo 23 questões; a escala de autoavaliação de síndrome de transtorno pré-menstrual de Steiner, que define o diagnóstico de TDPM, contendo 36 itens; e o Questionário de Autoavaliação da Escala de Hamilton para Depressão (QAEH-D), que foi validado para a versão brasileira, sendo composto por 18 itens $^{5,8,9}$.

Para a definição da presença ou ausência de TDPM na escala de Steiner, foram seguidos os critérios listados no Diagnostic and Statistical Manual of Mental Disorders - Fourth Edition (DSM IV), de acordo com o qual é obrigatória a presença de cinco ou mais sintomas nos dois últimos ciclos menstruais e é necessária a presença de pelo menos um dos sintomas relatados nos itens $1,2,3$ e 4 (Tabela 1$)^{5}$. 
Tabela 1 - Critérios da DSM IV para o diagnóstico do TDPM

1. Humor deprimido, sentimento de desesperança ou pensamentos de autodepreciação.

2. Ansiedade marcante, tensão, ou sentimento de estar "no limite".

3. Instabilidade afetiva marcante.

4. Raiva ou irritabilidade pronunciada, ou aumento significativo de conflitos pessoais.

5. Interesse diminuído nas atividades usuais.

6. Dificuldade de concentração.

7. Letargia, cansaço pronunciado, falta de energia habitual.

8. Mudança significativa no apetite, seja no sentido de diminuição ou de aumento.

9. Aumento ou diminuição importante no sono.

10. Sentimento de estar fora de controle.

11. Sintomas físicos, como dor mamária, dor de cabeça, dor muscular ou articular, ganho de peso.

DSM IV = Diagnostic and Statistical Manual of Mental Disorders - Fourth Edition; TDPM = transtorno disfórico pré-menstrual. Fonte: Steiner ${ }^{1}$.

Os sintomas de humor, depressivos e físicos do MSQ foram investigados separadamente. Para indicar a presença dos sintomas, considerou-se a marcação das alternativas de número 2 a 6 (2 - mínimo; 3 - suave; 4 - moderado; 5 - severo; e 6 - extremo), de modo que a marcação da alternativa nú- mero 1 (não aplicável, ausente, sem alterações) indicava que a entrevistada não apresentava os sintomas. Desta forma, foi possível classificar a voluntária como apresentando os sintomas se as respostas entre a categoria 2 e 6 correspondessem a $50 \%$ ou mais das respostas em cada categoria (Tabela 2$)^{8}$.

Tabela 2 - Critérios de classificação na Escala de Sintomas Menstruais (MSQ)

\begin{tabular}{|c|c|c|}
\hline Item & Categoria & Características \\
\hline $1,3,4,6,13,14,15,18$ e 21 & Humor e cognição & Riso; choro; estresse, irritabilidade; dificuldade de concentrar-se; e esquecimento. \\
\hline $5,9,11,17,19,20$ e 22 & Sintomas depressivos & $\begin{array}{l}\text { Sente-se só; deprimida; mais sensível; intolerante; isolamento no próprio trabalho; } \\
\text { pouca vontade de falar ou locomover-se; ponto de vista pessimista; dificuldade } \\
\text { para dormir; perda do interesse por atividades habituais. }\end{array}$ \\
\hline $2,7,8,10,12,16$ e 23 & Sintomas físicos & $\begin{array}{l}\text { Mastalgia; desconforto abdominal; edema generalizado, cansaço físico; susceptível } \\
\text { a mudanças de temperatura; e dor. }\end{array}$ \\
\hline
\end{tabular}

E para a classificação de presença ou ausência de sintomas depressivos utilizada no QAEH-D, estabeleceu-se como ponto de corte o valor de 10 , indicando a presença de sintomas depressivos ${ }^{9}$.

Foi realizada uma análise descritiva e analítica para expor os resultados obtidos. A apresentação das variáveis mensuradas foi feita através de tabelas ou gráficos. E para análise das variáveis quantitativas foi aplicado o teste qui-quadrado ou o teste exato de Fisher quando o qui-quadrado não pudesse ser aplicado. Todas as conclusões foram tomadas ao nível de significância de 5\%. Os software utilizados foram o Excel 2000 e o SPSS versão $8.0^{10}$.

\section{Resultados}

O perfil da amostra revela que a idade média foi de $22,4 \pm 3,8$, sendo que a maioria, $75,7 \%(\mathrm{n}=196)$, tinha idade entre 20 e 25 anos; 85,3\% $(\mathrm{n}=221)$ das acadêmicas eram solteiras e $64,1 \%,(n=166)$ naturais da região Metropolitana de Recife. Boa parte da amostra, 80,3\% $(\mathrm{n}=208)$, relatou ter algum tipo de ocupação; destas, apenas 17,0\% $(\mathrm{n}=44)$ recebiam remuneração de $\mathrm{R} \$ 683,05 \pm 736,41$. Com relação à etnia, observou-se uma predominância de brancas, 58,7\% $(\mathrm{n}=152)$ das respondentes, e a maioria, $72,6 \%(\mathrm{n}=188)$, declarou ser católica (Tabela 3). 
Transtorno disfórico pré-menstrual em universitárias - CARVALHO ET AL.

Tabela 3 - Distribuição das acadêmicas do curso de Fisioterapia da Faculdade Integrada do Recife (PE) quanto aos dados sociobiodemográficos

\begin{tabular}{|c|c|c|}
\hline Dados sociobiodemográficos & $\mathbf{n}$ & $\%$ \\
\hline $\begin{array}{l}\text { Idade }(22,4 \pm 3,8) \\
\quad<20 \\
20 \text { a } 22 \\
23 \text { a } 25 \\
>25\end{array}$ & $\begin{array}{c}41 \\
116 \\
80 \\
22\end{array}$ & $\begin{array}{c}15,8 \\
44,8 \\
30,9 \\
8,5\end{array}$ \\
\hline $\begin{array}{l}\text { Período que está cursando } \\
1^{\circ} \text { ao } 3^{\circ} \text { período } \\
4^{\circ} \text { ao } 7^{\circ} \text { período }\end{array}$ & $\begin{array}{c}50 \\
146\end{array}$ & $\begin{array}{l}19,3 \\
56,4\end{array}$ \\
\hline $\begin{array}{l}\text { Situação conjugal } \\
\text { Casada/União consensual } \\
\text { Solteira } \\
\text { Outros }\end{array}$ & $\begin{array}{c}21 \\
221 \\
17\end{array}$ & $\begin{array}{c}8,1 \\
85,3 \\
6,6\end{array}$ \\
\hline $\begin{array}{l}\text { Naturalidade } \\
\text { Região metropolitana de Recife } \\
\text { Outras cidades }\end{array}$ & $\begin{array}{c}166 \\
93\end{array}$ & $\begin{array}{l}64,1 \\
35,9\end{array}$ \\
\hline $\begin{array}{l}\text { Possui ocupação } \\
\text { Sim } \\
\text { Não } \\
\text { Não responderam }\end{array}$ & $\begin{array}{c}208 \\
50 \\
1\end{array}$ & $\begin{array}{c}80,3 \\
19,3 \\
0,4\end{array}$ \\
\hline $\begin{array}{l}\text { Tem rendimento } \\
\text { Sim } \\
\text { Não } \\
\text { Não informou/Não sabe }\end{array}$ & $\begin{array}{c}44 \\
203 \\
12\end{array}$ & $\begin{array}{c}17,0 \\
78,4 \\
4,6\end{array}$ \\
\hline $\begin{array}{l}\text { Cor } \\
\text { Branca } \\
\text { Negra } \\
\text { Parda } \\
\text { Amarela } \\
\text { Indígena } \\
\text { Outros }\end{array}$ & $\begin{array}{c}152 \\
17 \\
63 \\
21 \\
4 \\
2\end{array}$ & $\begin{array}{c}58,7 \\
6,6 \\
24,3 \\
8,1 \\
1,5 \\
0,8\end{array}$ \\
\hline $\begin{array}{l}\text { Religião } \\
\text { Católica } \\
\text { Espírita } \\
\text { Evangélica } \\
\text { Outras }\end{array}$ & $\begin{array}{l}188 \\
20 \\
37 \\
14\end{array}$ & $\begin{array}{c}72,6 \\
7,7 \\
14,3 \\
5,4\end{array}$ \\
\hline
\end{tabular}

Em relação à menarca, encontrou-se que em 62,9\% $(\mathrm{n}=$ 163) dos casos ela ocorreu até os 12 anos de idade. A menstruação habitualmente tinha fluxo moderado em $71,4 \%(\mathrm{n}=$ $185) ; 58,7 \%(\mathrm{n}=152)$ das acadêmicas estavam no meio do ciclo. Quanto às atividades sexuais, 70,7\% $(\mathrm{n}=183)$ afirmaram ter vida sexual ativa, sendo que $59,6 \%(n=109)$ ini- ciaram a atividade antes de completarem 18 anos de idade; $94,5 \%(n=173)$ utilizam métodos contraceptivos, dentre os quais se sobressaiu a utilização da pílula - 60,7\% $(\mathrm{n}=105)$.

Foi observado no estudo que $74,6 \%(n=106)$ das acadêmicas apresentavam dor de coluna como a condição física associada mais prevalente (Figura 1). Dentre elas, 37,3\%

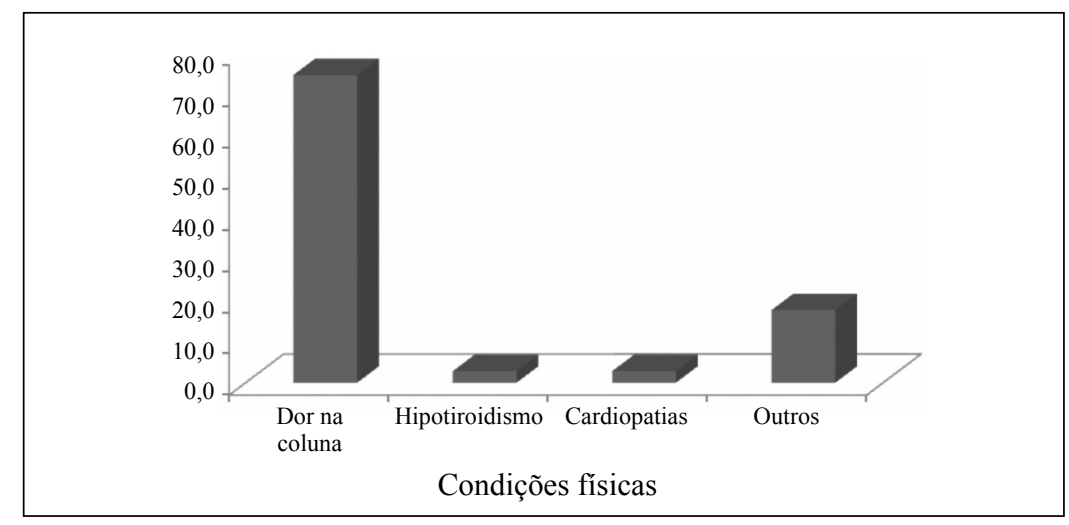

Figura 1 - Descrição das principais condições físicas associadas ao transtorno disfórico pré-menstrual entre as acadêmicas do curso de Fisioterapia da Faculdade Integrada do Recife, Recife (PE) 
$(\mathrm{n}=53)$ preocupavam-se de forma relativamente discreta com essa dor ou desconforto, 47,9\% $(n=68)$ das acadêmicas descreveram sentir certa dificuldade para lidar com essa sintomatologia, 43,7\% $(\mathrm{n}=62)$ confirmaram que a dor ou desconforto quase não as impedia de realizar as suas atividades cotidianas, e 34,5\% $(n=49)$ ficavam levemente cansadas devido a esse problema.
Ao serem pesquisados os principais sintomas físicos descritos, observamos que a intensidade de dor predominante foi a moderada de acordo com os seguintes sintomas: $25,5 \%(\mathrm{n}=$ 66) das acadêmicas apresentaram a sensação de peso abdominal associada com desconforto ou dor; em 22,8\% $(n=59)$ dos casos experimentaram dores intermitentes ou cólicas abdominais; $26,3 \%(\mathrm{n}=68)$ sentem uma sensação de inchaço; e $26,6 \%(\mathrm{n}=$ 69) ficam com as mamas túrgidas e inchadas (Figura 2).

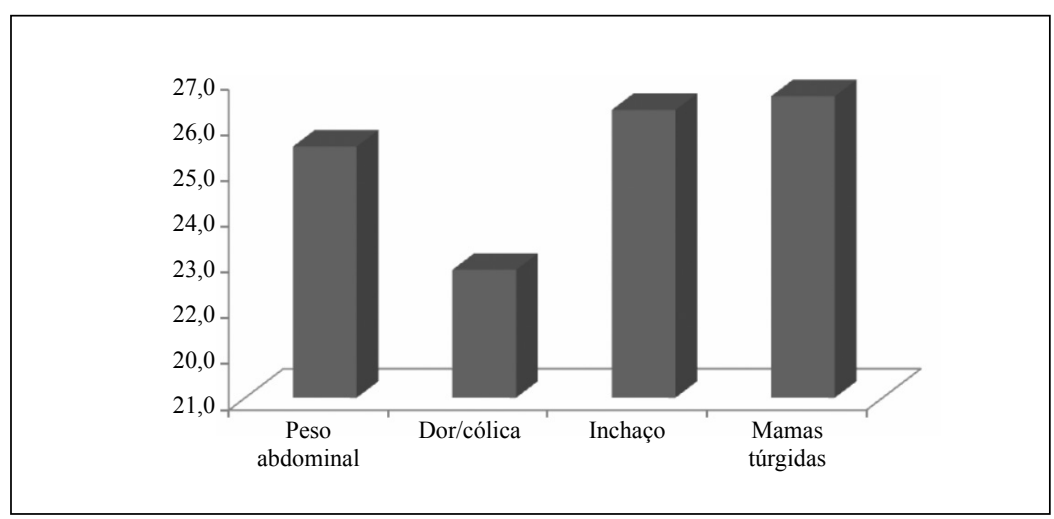

Figura 2 - Descrição dos principais sintomas físicos associados ao transtorno disfórico pré-menstrual entre as acadêmicas do curso de Fisioterapia da Faculdade Integrada do Recife, Recife (PE)

$\mathrm{Na}$ amostra estudada foi encontrada a prevalência de 6,2\% $(\mathrm{n}=16)$ de acadêmicas portadoras de TDPM. Quando correlacionamos os antecedentes pessoais e as comorbidades, pode-se observar que o fato de as acadêmicas já apresentar problemas emocionais esteve associa- do à presença de TDPM $(\mathrm{p}=0,03)$; entretanto, as demais variáveis pesquisadas, como utilização de medicamentos, antecedente familiar e presença de comorbidades, não revelaram nenhum associação significativa com o TDPM (Tabela 4).

Tabela 4 - Distribuição das acadêmicas do curso de Fisioterapia da Faculdade Integrada do Recife com transtorno disfórico pré-menstrual e a sua correlação com os antecedentes pessoais, antecedentes hereditários e doenças

\begin{tabular}{lcc}
\hline & \multicolumn{1}{c}{ TDPM } \\
Antecedentes pessoais, antecedentes hereditários e doenças & Não, n (\%) & Sim, n (\%) \\
\hline Usando algum medicamento prescrito por médico & p \\
Sim & $78(32,1)$ & $6(37,5)$ \\
Não & $165(67,9)$ & $10(62,5)$ \\
Medicamento utilizado & & 0,783 \\
Anticoncepcional & $49(62,8)$ & $5(83,3)$ \\
Psicofármacos & $14(17,9)$ & $1(16,7)$ \\
Outros & $17(21,8)$ & $1(16,7)$ \\
Tratamento para problemas emocionais & & - \\
Sim & $27(11,1)$ & $5(31,3)$ \\
Não & $216(88,9)$ & $11(68,8)$ \\
Na família alguém fez ou faz tratamento por problemas emocionais & & 0,034 \\
Sim & $83(34,2)$ & $8(50,0)$ \\
Não & $160(65,8)$ & $8(50,0)$ \\
Doenças & \multicolumn{2}{c}{0,279} \\
Diabetes & $1(0,8)$ & $0(0,0)$ \\
Hipertensão arterial & $2(1,5)$ & $0(0,0)$ \\
Cardiopatias & $2(1,5)$ & $0(0,0)$ \\
Hipotiroidismo & $4(3,1)$ & $0(0,0)$ \\
Dor na coluna & $94(72,3)$ & $12(100,0)$ \\
Outros & $25(19,2)$ & $0(0,0)$ \\
Não sabem informar & $2(1,50)$ & $0(0,0)$ \\
\hline
\end{tabular}

TDPM = transtorno disfórico pré-menstrual. 
Quando utilizamos os sintomas constantes no MSQ e os relacionamos com a presença de TDPM, revela-se uma associação estatisticamente significante desse transtorno com a depressão $(\mathrm{p}=0,048)$. Os demais sintomas como ansiedade, labilidade de humor, sintomas somáticos, habilidades e dor não se apresentaram estatisticamente significantes quando correlacionados ao TDPM (Tabela 5).

Tabela 5 - Distribuição das acadêmicas do curso de Fisioterapia da Faculdade Integrada do Recife com transtorno disfórico pré-menstrual e a sua correlação com as escalas do Questionário de Sintomas Menstruais

\begin{tabular}{|c|c|c|c|}
\hline \multirow[b]{2}{*}{ Escala do MSQ } & \multicolumn{3}{|c|}{ TDPM } \\
\hline & Sim, n (\%) & Não, n (\%) & $\mathbf{p}$ \\
\hline \multicolumn{4}{|l|}{ Depressão } \\
\hline Sim & $16(100,0)$ & $195(80,2)$ & \\
\hline Não & $0(0,0)$ & $48(19,8)$ & 0,048 \\
\hline \multicolumn{4}{|l|}{ Ansiedade } \\
\hline Sim & $16(100,0)$ & $225(92,6)$ & \\
\hline Não & $0(0,0)$ & $18(7,4)$ & 0,612 \\
\hline \multicolumn{4}{|l|}{ Labilidade do humor } \\
\hline Sim & $16(100,0)$ & $209(86,0)$ & \\
\hline Não & $0(0,0)$ & $34(14,0)$ & 0,141 \\
\hline \multicolumn{4}{|c|}{ Sintomas sintomáticos } \\
\hline Sim & $16(100,0)$ & $233(95,9)$ & \\
\hline Não & $0(0,0)$ & $10(4,1)$ & 1,000 \\
\hline \multicolumn{4}{|l|}{ Habilidades } \\
\hline Sim & $15(93,8)$ & $218(89,7)$ & \\
\hline Não & $1(6,3)$ & $25(10,39)$ & 1,000 \\
\hline \multicolumn{4}{|l|}{ Dor } \\
\hline Sim & $16(100,0)$ & $216(88,99)$ & \\
\hline Não & $0(0,0)$ & $27(11,1)$ & 0,388 \\
\hline
\end{tabular}

MSQ = Questionário de Sintomas Menstruais; TDPM = transtorno disfórico pré-menstrual.

\section{Discussão}

Em outros estudos conduzidos no Brasil, foi possível observar um perfil das variáveis socioeconômicas e demográficas semelhante ao da presente pesquisa, na qual as acadêmicas eram em sua maioria solteiras, encontravam-se em idade reprodutiva e tinham algum tipo de ocupação $0^{11-13}$.

No presente estudo a menarca foi mais prevalente na faixa etária de 12 anos de idade, assim como o início da vida sexual se localizou predominantemente na faixa etária abaixo dos 18 anos. Em um estudo realizado com adolescentes na cidade de Santo André (SP) por Azevedo et al. ${ }^{14}$, as jovens que menstruaram mais cedo também relataram o início de sua atividade sexual de forma precoce. E de acordo com Veras \& $\mathrm{Nardi}^{15}$, a precocidade na ocorrência da menarca favorece a maior incidência de sintomas depressivos entre as mulheres.

É importante lembrar que o TDPM se caracteriza como um transtorno de humor presente na fase lútea tardia do ciclo reprodutivo, fase em que as mulheres apresentam respostas imunológicas e neuroendócrinas ao estresse. Isso sugere que as alterações hormonais nesse momento do ciclo menstrual podem contribuir para o desenvolvimento de sintomas do TDPM, e na nossa amostra a maioria das acadêmicas se encontravam em idade fértil ${ }^{15,16}$.
Para o controle da natalidade foi observado um elevado uso de metódos contraceptivos entre as acadêmicas, sobressaindo-se a pílula anticoncepcional. Sabe-se que os ciclos anovulatórios poderiam resultar na redução dos sinais e sintomas prevalentes na síndrome da TPM, mas não acontece o mesmo com as pacientes diagnosticadas com TDPM. Antunes et al. ${ }^{17}$ concluíram em seu estudo, realizado na Universidade Paulista em Bauru (SP), que os transtornos psiquiátricos ligados à variação pré-menstrual não parecem melhorar com o uso de pílulas anticoncepcionais. Já Bianco et al. ${ }^{18}$ sugerem que o uso de anticoncepcionais pode estar relacionado com os graus mais elevados da depressão em mulheres predispostas a este sintoma.

A comorbidade mais relatada entre as acadêmicas foi a dor lombar (lombalgia), e no estudo realizado por Muramatsu et al. ${ }^{11}$, observou-se que a presença de dores se encontra em primeiro lugar dentre os sintomas físicos mais incidentes nas portadoras de TPM, sugerindo que os sintomas físicos se apresentam como queixa clínica tanto em mulheres portadoras de TPM como naquelas com diagnóstico de TDPM.

$\mathrm{Na}$ amostra estudada foi encontrada a prevalência de $6,2 \%$ de acadêmicas portadoras de TDPM, assemelhando-se ao visto por da Silva ${ }^{19}$ em estudo de coorte de base populacional onde foram utilizados os mesmos critérios do DSM IV 
para a classificação da TDPM. Neste estudo, pode-se observar uma prevalência de 5,8\% para TDPM, sendo que, após 20 meses de acompanhamento na mesma população, a incidência cumulativa foi de $7,4 \%$.

Um número cada vez maior de mulheres em idade reprodutiva vem verbalizando as repercussões físicas e emocionais da TPM, mas os sintomas de ordem emocional têm sido referidos como de maior intensidade. Este fato também é apontado em outros trabalhos que avaliaram os sintomas prémenstruais e suas repercussões no cotidiano das mulheres, enfatizando que os sintomas de alteração de humor podem provocar prejuízo ou mesmo necessidade de interrupção dos compromissos, gerando problemas no relacionamento familiar e social, bem como dificuldades no desempenho acadêmico e/ou profissional ${ }^{1,3,7,14}$.

Uma hipótese que pode ser levantada é a de que os quadros disfóricos pré-menstruais representam uma sequela de um episódio depressivo anterior ou, alternativamente, uma forma precursora de um episódio depressivo que mais tarde irá se desenvolver ${ }^{20}$.

As alterações nos níveis de estrogênio e progesterona explicam alguns sintomas de TDPM nos quais a falta de estrogênio pode estar relacionada ao aparecimento da depressão, com diminuição da atividade psicomotora, e seus níveis elevados podem estar associados a estados disfóricos como agitação, ansiedade e irritabilidade, os quais se mostraram presentes em todas as acadêmicas que apresentam TDPM ${ }^{21}$.

Atualmente, a depressão apresenta-se como uma doença que afeta muitas pessoas. Segundo Campos ${ }^{22}$, a estimativa de pessoas depressivas na população varia de 10 a $15 \%$; entre as mulheres, esse índice sobe para $20 \%$. No presente estudo, pode-se observar uma correlação positiva entre os sintomas depressivos e o TDPM. Esses dados sugerem que este possa ser um subtipo clínico de transtorno depressivo. Isto confirma os resultados obtidos no estudo de Veras \& $\mathrm{Nardi}^{15}$, que demonstraram que a depressão é uma variável clínica consistentemente relacionada com o TDPM.

\section{Conclusão}

No presente estudo, foi encontrada uma alta prevalência de sintomas somáticos associados ao TDPM, mas, como foi visto, eles não interferem de forma significativa nas atividades cotidianas das acadêmicas. No entanto, entre os sintomas emocionais, os sintomas depressivos mostraram uma correlação positiva com os critérios de diagnóstico do TDPM utilizados na presente pesquisa.

O TDPM revelou uma correlação significativa com os sintomas depressivos, sugerindo tratar-se de uma expressão clínica de transtorno de humor.

\section{Referências}

1. Steiner M. Premenstrual syndrome and premenstrual dysphoric disorder: guidelines for management. J Psychiatry Neurosci. 2000;25(5):459-68.

2. Valadares GC, Ferreira LV, Filho HC, Romano-Silva MA. Transtorno disfórico pré-menstrual revisão - conceito, história, epidemiologia e etiologia. Rev Psiquiatr Clin. 2006;33(3):117-23.

3. de Andrade LH, Viana MC, Silveira CM. Epidemiologia dos transtornos psiquiátricos na mulher. Rev Psiquiatr Clin. 2006;33(2):43-54.

4. Vieira Filho AH, Tung TC, Artes R. Escalas de avaliação de transtorno prémenstrual. Rev Psiquiatr Clin. 1998;25(5):273-8.

5. Freeman EW, Sondheimer SJ. Premenstrual dysphoric disorder: recognition and treatment. primary care companion. J Clin Psychiatry. 2003;5(1):30-9.

6. Braverman PK. Premenstrual syndrome and premenstrual dysphoric disorder. J Pediatr Adolesc Gynecol. 2007;20(1):3-12.

7. Pires ML, Calil HM. Associação entre transtorno disfórico pré-menstrual e transtornos depressivos. Rev Bras Psiquiatr.1999;21(2):118-27.

8. Webster SK, Martin HJ, Uchalik D,Gannon L. The Menstrual Symptom Questionnaire and spasmodic/congestive dysmenorrhea: measurement of an invalid construct. J Behav Med. 1979;2(1):1-19.

9. Moraes JL, Ximenes RC, dos Passos MP, Sougey EB. Validação da versão brasileira do Questionário de Auto-Avaliação da Escala de Hamilton para Depressão (QAEHD) [tese]. Recife: Universidade Federal de Pernambuco; 2008.

10. Jerrold HZ. Biostatistical analysis. 3rd ed. New Jersey: Prentice Hall; 1996.

11. Muramatsu CH, Vieira OC, Simões CC, Katayama DA, Nakagawa FH. Conseqüências da síndrome da tensão pré-menstrual na vida da mulher. Rev Esc Enferm USP. 2001;35(3):205-13.

12. Rodrigues IC, de Oliveira E. Prevalência e convivência de mulheres com síndrome pré-menstrual. Arq Cienc Saude. 2006;13(3):146-52.

13. Wang YP, Teng CT, Vieira Filho AH, Gorenstein C, Andrade LH. Dimensionality of the premenstrual syndrome: confirmatory factor analysis of premenstrual dysphoric symptoms among college students. Braz J Med Biol Res. 2007;40(5):639-47.

14. de Azevedo MR, Saito MI, Berenstein E, Viegas D. Síndrome pré-menstrual em adolescentes: um estudo transversal dos fatores biopsicossociais. Arq Med ABC. 2006;31(1):12-7.

15. Veras AB, Nardi AE. Hormônios sexuais femininos e transtornos do humor. J Bras Psiquiatr. 2005;54(1):57-68.

16. Born L, Phillips SD, Steiner M, Soares CN. Trauma e o ciclo reprodutivo feminino. Rev Bras Psiquiatr. 2005;27(Supl II):S65-72.

17. Antunes G, Rico VV, Gouveira Junior A. Variações da ansiedade relatada em função do ciclo menstrual e do uso de pílulas anticoncepcionais. Interacao Psicol. 2004;8(1):81-7.

18. Bianco SM, Barancelli L, Roveda AK, Santin JC. Influência do ciclo menstrual em episódios depressivos. Arq Cienc Saude UNIPAR. 2004;8(1):11-7.

19. da Silva CM. Síndrome pré-mestrual: prevalência e fatores associados na população urbana de Pelotas [dissertação]. Pelotas: Universidade Federal de Pelotas; 2004.

20. Cheniaux E. Tratamento da disforia pré-menstrual com antidepressivos: revisão dos ensaios clínicos controlados. J Bras Psiquiatr. 2006;55(2):142-7.

21. Mendonça Lima CA, Camus V. Síndrome pré-menstrual: um sofrimento ao feminino. Psiquiatr Biol. 1996; 4(3):137-46.

22. Campos R. Depressão, um estigma feminino. Rev Viver Psicol. 2002;10(112):24-9. 